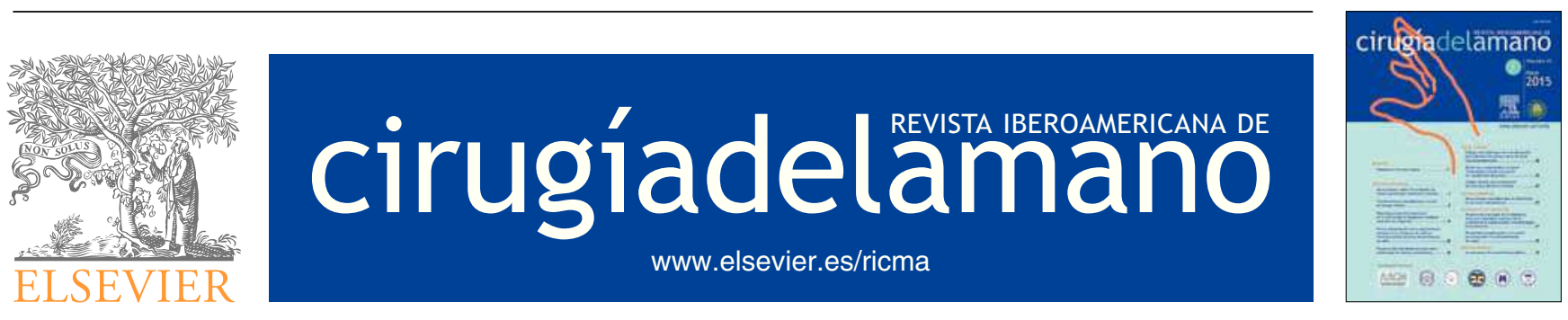

ARTÍCULO ORIGINAL

\title{
Pseudoartrosis y consolidaciones viciosas de falanges distales
}

\author{
A. Ferreres ${ }^{\mathrm{a} *}$, M.A. Gómez ${ }^{\mathrm{b}}$, R. Gutiérrez ${ }^{\mathrm{c}}$ \\ a Institut Kaplan. Cirugía de la Mano. Barcelona. \\ b Unidad de Cirugía de la Mano. Hospital Pablo Tobon Uribe. Medellín, Colombia. \\ c Instituto Traumatológico. Instituto de Seguridad del Trabajador. Santiago, Chile.
}

Recibido el 29 de septiembre de 2014. Aceptado el 9 de febrero de 2015.

\section{PALABRAS CLAVE \\ Pseudoartrosis; \\ Falange distal; \\ Mala unión}

\section{KEYWORDS}

Non-union;

Distal phalanx;

Mal-union

\begin{abstract}
Resumen
Objetivo: Analizar los resultados obtenidos en el tratamiento quirúrgico de las pseudoartrosis y mala consolidación de falange distal.

Material y método: Se revisaron las historias clínicas de tres pacientes que fueron tratados de una pseudoartrosis y uno de una mala consolidación de la falange distal. El tratamiento consistió en resecar el tejido fibroso, realizar un aporte de hueso esponjoso autólogo y una fijación interna con tornillos de minifragmentos. La vía de acceso varió en función de la localización de la lesión.

Resultados: En todos los pacientes se obtuvo la consolidación y reanudaron sus actividades previas sin molestias.

Conclusión: La pseudoartrosis y/o mala consolidación de falange distal puede solucionarse de forma efectiva mediante un apropiado abordaje quirúrgico, una fijación interna estable y aporte de injerto óseo autólogo.

๑ 2015, SECMA. Publicado por Elsevier España, S.L.U. Este es un artículo Open Acces distribuido bajo los términos de la licencia CC BY-NC-ND (http://creativecommons.org/licenses/by-nc-nd/4.0/).
\end{abstract}

Non-union and mal-union of the distal phalanx

\begin{abstract}
Purpose: To analyze the results of surgical treatment of non-unions and mal-unions of the distal phalanx.

Material and method: The clinical records of four patients were analyzed. Three were treated for a non union and one for a mal union of the distal phalanx. In all of them, the treatment consisted in resection of the fibrous tissue, bone graft from the distal radius, and fixation with mini-fragment screws. The approach depended on the location of the lesion.

Results: In all cases the consolidation was achieved and patients returned to their previous activities.
\end{abstract}

*Autor para correspondencia.

Correo electrónico: angelferreres@institut-kaplan.com.

(c) 2015, SECMA. Publicado por Elsevier España, S.L.U. Este es un artículo Open Acces distribuido bajo los términos de la licencia CC BY-NC-ND (http://creativecommons.org/licenses/by-nc-nd/4.0/). 
Conclusion: Non-union and mal-union of distal phalanx can be effectively treated through an adequate approach that will depend on the site of the lesion, bone graft and a stable internal fixation.

(c) 2015, SECMA. Published by Elsevier España, S.L.U. This is an open access article under the CC BY-NC-ND license (http://creativecommons.org/licenses/by-nc-nd/4.0/).

\section{Introducción}

Las fracturas de la falange distal son las fracturas más frecuentes de la mano ${ }^{1}$. La gran mayoría de ellas son tratadas de forma conservadora sin presentar grandes tasas de complicaciones como las malas consolidaciones, pseudoartrosis o distrofias ungueales.

Es poco frecuente la no consolidación. Jupiter y cols. ${ }^{2}$ realizaron una revisión de las fracturas de metacarpianos y falanges encontrando que la gran mayoría de estas fracturas presenta una consolidación "clínica" entre la tercera y sexta semanas, pero que los signos radiológicos de consolidación pueden tardar hasta cuatro meses en aparecer. Por ello consideramos que se define como no consolidación ósea aquella fractura de falange distal que tras más de 4 meses de evolución, persiste con movilidad y dolor en el foco de fractura y no presenta signos radiológicos de consolidación.

En el presente artículo se presentan tres casos de pseudoartrosis y una mala unión de falange distal. Se ha realizado una revisión de la literatura, y se propone un esquema de tratamiento.

\section{Material y método}

Se han revisado 4 pacientes tratados en nuestro centro, que corresponden a 3 casos de pseudoartrosis, (uno en la parte media de la falange, uno de la parte distal y uno base de falange) y una mala consolidación de la base.

\section{Caso 1}

Paciente diestro de 31 años, que 6 meses antes había sufrido un traumatismo escalando sobre el dedo índice de su mano izquierda. Radiológicamente se apreció una pseudoartrosis mal alineada del tercio medio de la falange distal. Se realizó una incisión en boca de pez, pero situando el trazo transversal en el límite del hiponiquio exponiendo completamente la falange distal protegiendo las estructuras del aparato ungueal (fig. 1). Se practicó un avivamiento de los extremos de la pseudoartrosis, un aporte de esponjosa procedente del extremo distal del radio y una osteosíntesis con un minitornillo de $2 \mathrm{~mm}$ de diámetro compact hand (DePuySynthes, West Chester, PA, EE.UU.), a compresión, colocado de distal a proximal (fig. 2).

Se obtuvo la consolidación radiológica a los 2 meses de seguimiento, estando el paciente asintomático en la última revisión, al año, y con una movilidad de la interfalángica distal (IFD) de $0^{\circ} / 55^{\circ}$.

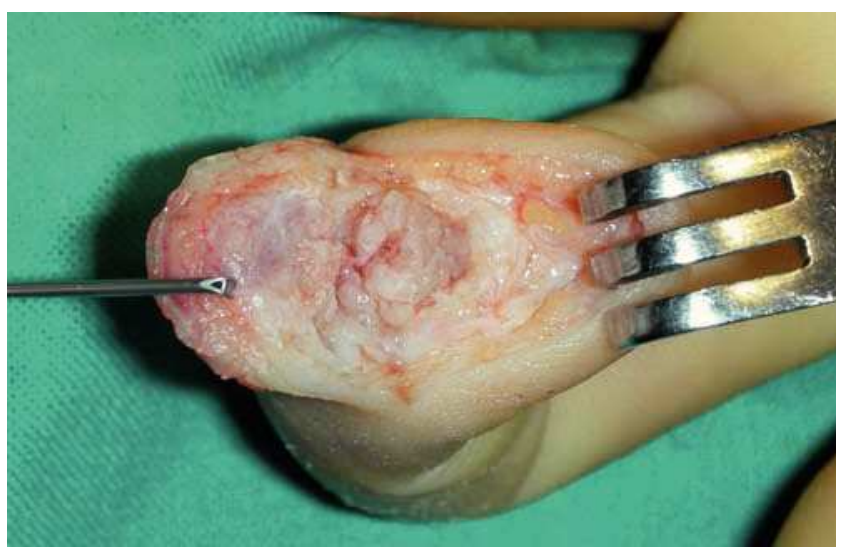

Figura 1 Acceso realizado en el caso 1. Nótese que la parte transversal de la incisión se sitúa justo en el límite del lecho ungueal.

\section{Caso 2}

Paciente diestro de 47 años, cazador de afición que 13 meses antes había sufrido un corte con una sierra radial en el pulpejo del índice de la mano izquierda, con una pseudoartrosis de la falange distal con síntomas de inestabilidad al realizar pinza y al apretar el gatillo (dispara con la izquierda).

Se realizó, en este caso, un acceso dorsal entre la inserción del tendón extensor y la parte proximal de la matriz ungueal, exponiendo el foco de pseudoartrosis. Se avivaron los extremos óseos de la falange y se realizó la osteosíntesis con dos tornillos de minifragmentos de $1 \mathrm{~mm}$ de diámetro compact hand (DePuy-Synthes, West Chester, PA, EE.UU.) cruzados, obteniendo una adecuada estabilidad. A los dos meses tras comprobar la consolidación ósea, el paciente retomó su afición sin problemas y con una movilidad de la IFD de $0^{\circ} / 45^{\circ}$.

\section{Caso 3}

Paciente cirujano cardíaco diestro de 30 años que había sufrido un traumatismo sobre el dedo meñique de su mano derecha 2 años antes. Refería dolor en la práctica de su profesión al realizar nudos. Se observó una pseudoartrosis de la parte distal de la falange y fue intervenido a través de un acceso en boca de pez, aportando injerto y una osteosíntesis con un tornillo compact hand (DePuy-Synthes, West Chester, PA, EE.UU.). En este caso la consolidación obtenida fue parcial (fig. 3), pero con una estabilidad completa, y el paciente desempeña su profesión sin molestias 10 años después de la intervención. 

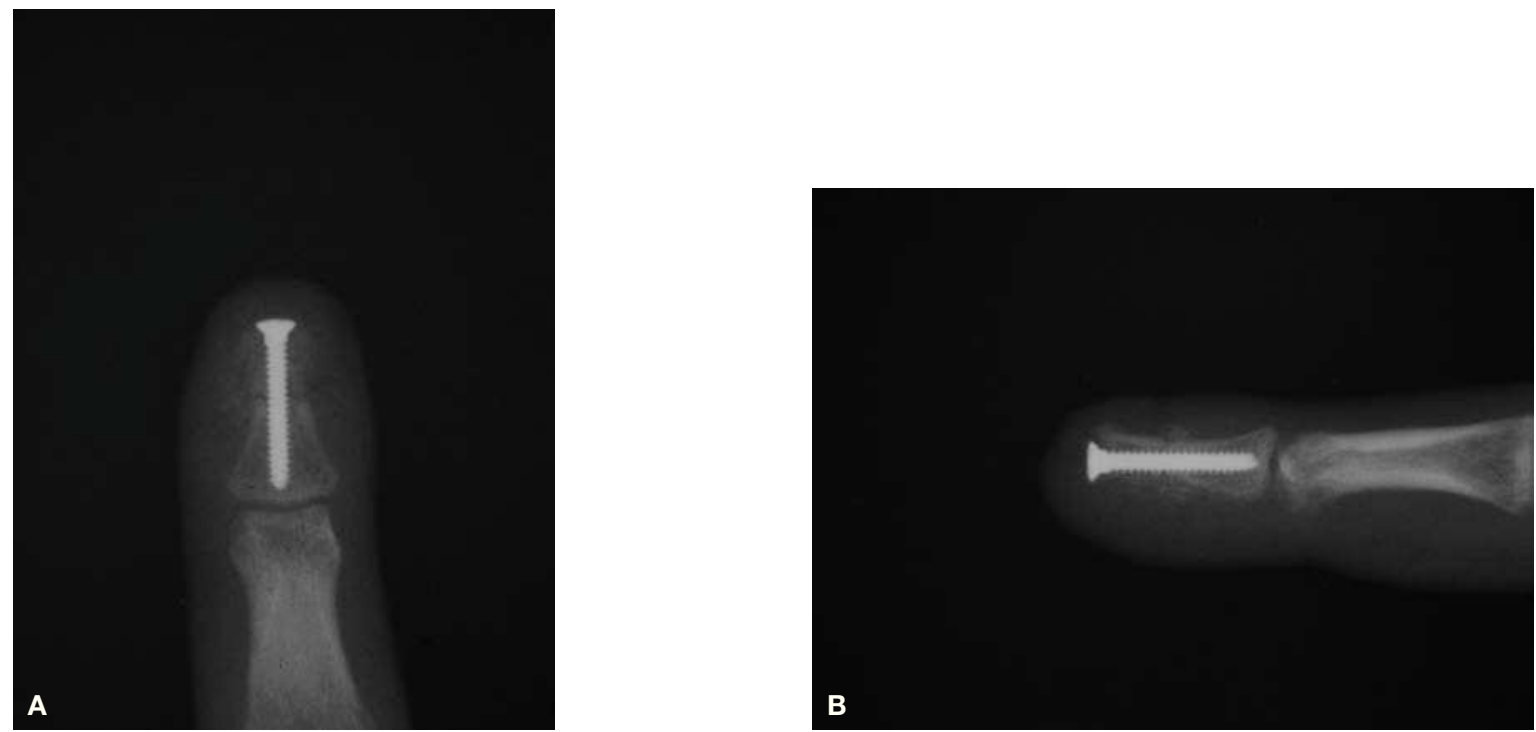

Figura 2 (A) Proyección anteroposterior de la falange distal del caso 1 al que se colocó un tornillo ya que la parte proximal permitía un agarre suficiente. Se observan fragmentos del injerto utilizado proveniente de la epífisis distal del radio. (B) Proyección lateral del mismo caso.

\section{Caso 4}

Se trató de un paciente diestro, de 34 años de edad, que 10 meses antes había sufrido un accidente de motocicleta y que refería molestias a la realización de la pinza con el dedo medio de su mano izquierda. Radiológicamente se observó una deformidad en extensión de la parte proximal de la falange distal superior a $30^{\circ}$ (fig. 4A).

Por vía dorsal se realizó una osteotomía correctora entre la inserción del tendón extensor y el inicio de la matriz ungueal que se fijó con dos tornillos de $1 \mathrm{~mm}$ compact hand (DePuy-Synthes, West Chester, PA, EE.UU.) que consolidó sin incidencias a las 8 semanas (fig. $4 \mathrm{~B}$ y $4 \mathrm{C}$ ) y presentando una movilidad de $0^{\circ} / 75^{\circ}$ en la IFD a los 5 meses.

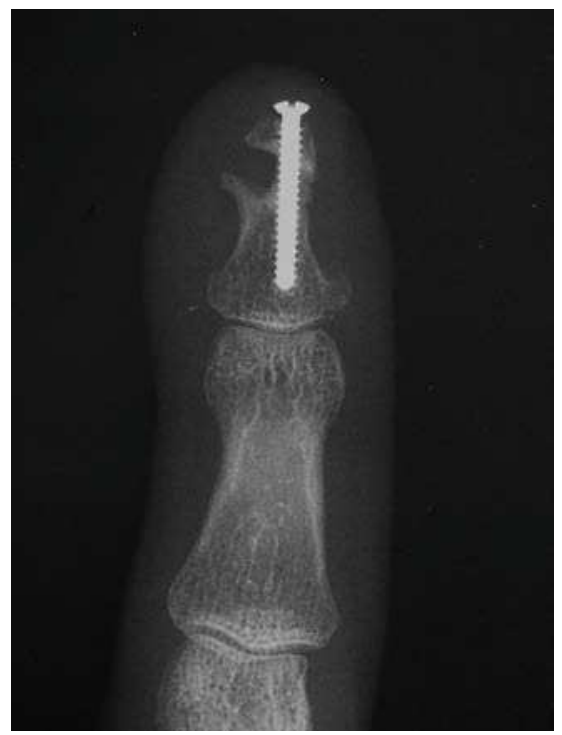

Figura 3 Aspecto de la consolidación parcial del caso 3.
Como complicaciones cabe mencionar la consolidación parcial en la pseudoartrosis de la parte distal de la falange y se observa por transparencia la coloración de la cabeza del tornillo a través del extremo distal del lecho ungueal.

\section{Discusión}

Las fracturas de la falange distal se dividen en: fracturas del extremo distal, de la diáfisis y de la base ${ }^{3}$. Las fracturas del extremo distal raramente presentan complicaciones y aunque su consolidación ocasionalmente pueda evolucionar a una pseudoartrosis, esta raramente es sintomática ${ }^{1}$. Las fracturas de la diáfisis son las que más riesgo de pseudoartrosis presentan, por sus características anatómicas y por estar sometidas a mayores cargas que otras zonas ${ }^{2}$. Las fracturas de la base son generalmente el resultado de avulsiones de los tendones flexores o extensores. Su complicación más frecuente es la mala consolidación y mala reducción articular con la subsiguiente deformidad y alteración del arco de movilidad articular ${ }^{4}$.

Se han identificado unos factores de riesgo que predisponen al desarrollo de una pseudoartrosis de la falange distal $^{5}$ :

1. Lesión importante de tejidos blandos circundantes

2. Alta energía (Aplastamiento o amputación)

3. Compromiso vascular

4. Infección

5. Pérdida ósea segmentaria

6. Inmovilización inadecuada

En la literatura consultada no existe acuerdo respecto a cuántas de las fracturas de las falanges distales evolucionan a una pseudoartrosis. Desde $\mathrm{DaCruz}^{6}$ que encontró tasas de no consolidación del $47 \%$ tras 6 meses de seguimiento, hasta 

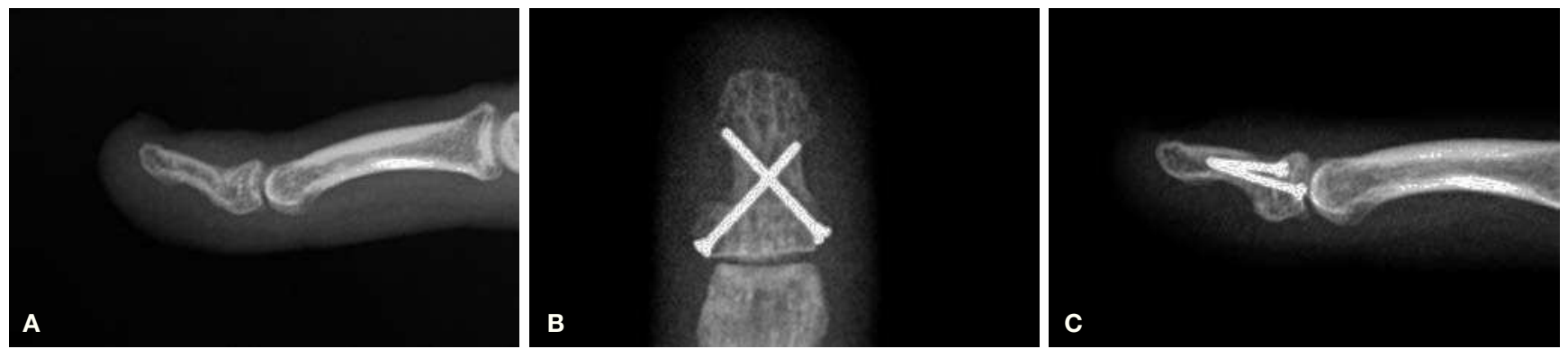

(A) Angulación dorsal de una mala unión de la falange distal correspondiente al caso 4. (B) Se observa la colocación de los dos tornillos de minifragmentos de $1 \mathrm{~mm}$ de diámetro entrados desde la parte lateral de la base de la falange. Los tornillos tienen un buen agarre en la cortical opuesta. (C) Proyección lateral del mismo caso.

Barton $^{7}$ que, en su revisión, encontró tasas de no unión para los metacarpianos del $0,7 \%$ y para las falanges del $0,2 \%$, aunque incluía a todas las falanges en la revisión. Van Oosterom ${ }^{8}$ reportó un $6 \%$ de pseudoartrosis en fracturas de falanges tratadas quirúrgicamente. A pesar de una aparente baja prevalencia en algunas de las series, la no consolidación genera una considerable incapacidad para realizar labores de la vida diaria en el $70 \%$ de los pacientes ${ }^{5}$.

En pacientes poco sintomáticos, puede proponerse un tratamiento conservador y, en función de la evolución y localización del foco de pseudoartrosis, realizar la exéresis del fragmento distal de la falange si ese es de pequeño tamaño y no compromete la longitud del dedo².

En los pacientes que presentan síntomas repetitivos de inestabilidad en el foco de fractura y molestias para realizar la pinza digital debe considerarse realizar un tratamiento quirúrgico. El tratamiento no diferirá del aplicado a otras localizaciones y consistirá en la exposición de la pseudoartrosis, el avivamiento de los extremos óseos con un aporte de injerto, esponjoso generalmente y osteosíntesis. En el caso de una mala consolidación, la osteotomía será el tratamiento indicado con la osteosíntesis adecuada. Quizá sea este último aspecto el que merezca una discusión más interesante. Además de la técnica de osteosíntesis, el acceso quirúrgico constituye un aspecto técnico importante a discutir.

Itoh y cols. ${ }^{9}$ presentan una serie de casos de 6 pacientes con una no unión de la diáfisis a los que realizaron un abordaje longitudinal en el pulpejo; posteriormente avivaron los bordes de los fragmentos de la falange y aplicaron un injerto óseo extraído del cúbito proximal o del radio distal que estabilizaron con dos agujas de Kirschner cruzadas. Obtuvieron la consolidación radiológica en todos los casos entre la $6 .^{a}$ y la $11 .^{a}$ semana sin molestias cicatriciales o neurovasculares y con un adecuado rango de movilidad de la interfalángica distal en 5 de los 6 pacientes.

Chim y cols. ${ }^{10}$ posteriormente publicaron una serie de 14 casos con pseudoartrosis de la parte distal, diáfisis u oblicuas, a los que se les realizó un abordaje a través del lecho ungueal, y una fijación con minitornillos corticales. En todos los pacientes se obtuvo la consolidación, pero en 8 de los 14 esta tardó más de tres meses. En ninguno de los pacientes se utilizó injerto óseo y el mayor acortamiento fue del $30 \%$ comparado con el dedo sano. El arco de movilidad de la IFD no se alteró de forma importante obteniendo una media de $56^{\circ}$. Las molestias derivadas del material prominente se eli- minaron en todos los pacientes al retirar los tornillos de fijación.

Kim y cols. ${ }^{11}$ presentaron un método de fijación y aporte óseo en 13 pacientes con una pseudoartrosis de la falange distal a los que realizaron un abordaje en la punta del dedo, labrando el canal endomedular, y les insertaron retrógradamente una astilla de cortical tomada del cúbito proximal, radio distal o del ilíaco, que actuaba tanto de aporte óseo como de fijación. De hecho, este método había sido publicado por Shuinomiya unos años antes y publicado en una carta al editor ${ }^{12}$. En 3 pacientes se requirió una estabilización adicional con agujas de Kirschner. En todos los pacientes se obtuvo la consolidación ósea radiológica entre la $6 .^{\mathrm{a}}$ y la $8 .^{\mathrm{a}}$ semana. No aparecieron inestabilidades ni alteraciones ungueales. La movilidad mejoró significativamente así como el dolor preoperatorio en 10 de los 13 pacientes, persistiendo en los pacientes que previamente tenían una artrosis postraumática.

Como vemos, en las series en que no se utilizó injerto, la consolidación se retrasó y en la que no se usó material de osteosíntesis, se debió añadir posteriormente en 3/13 casos. Nosotros preferimos utilizar ambas técnicas. Özçelit y cols. ${ }^{13}$ usaron el cúbito proximal como fuente de injerto y usaron agujas de Kirschner como fijación y obtuvieron la consolidación en todos los casos.

Aunque autores como Voche y cols. ${ }^{14}$ no hayan encontrado diferencias entre la síntesis con agujas de Kirschner y con tornillos, nosotros, de la misma manera que Chim y cols. ${ }^{10}$ y Botelheiro ${ }^{15}$ nos inclinamos por un tornillo de minifragmentos retrógrado en el caso de que la parte proximal de la falange tenga la longitud suficiente para el agarre del tornillo. Si el fragmento proximal es pequeño preferimos entonces utilizar dos tornillos del menor diámetro posible introducidos desde cada lado de la base de la falange hasta la cortical opuesta del fragmento distal. Así no interferimos con los ligamentos ni con el tendón extensor ni con la matriz ungueal. En algún caso puede ser necesaria la resección ungueal para poder levantar en bloque matriz y lecho al abordar el foco de pseudoartrosis. La uña puede reponerse al final del procedimiento para evitar perjuicios estéticos.

En nuestros casos, de los factores de riesgo enumerados en la introducción, la inmovilización inadecuada había ocurrido en todos los casos.

Respecto al acceso quirúrgico de la lesión, creemos que si esta se encuentra distal a la inserción del FDP, el acceso 
recomendado es similar a la boca de pez pero pasando la parte transversal de la incisión entre hiponiquio y epidermis normal. De esta forma no quedan secuelas estéticas como en la boca de pez tradicional, motivo por el que no se aconseja su uso. De esta manera, no se ocasiona ninguna alteración al lecho ungueal y se preservan completamente la vascularización de los fragmentos y la inervación del pulpejo. En el caso de que la lesión a corregir se encuentre más proximal, recomendamos un acceso dorsal. El abordaje de la lesión se realizará entre la inserción del tendón extensor y el inicio de la matriz ungueal. Es la única parte del dorso de la falange que queda libre de estructuras importantes. La osteosíntesis puede realizarse mediante dos tornillos, del menor calibre posible, cruzados y entrando desde los lados de la base de la falange (fig. 5).

\section{Conclusión}

Si bien las fracturas de la falange distal son las fracturas más frecuentes de la mano, pocas veces presentan una pseudoartrosis sintomática.

El paciente con una pseudoartrosis o mala consolidación de una fractura de falange distal refiere un traumatismo previo que, generalmente, fue tratado defectuosamente, bien por no buscar consejo médico, por una inmovilización de corta duración, o por un tratamiento inadecuado. Otros factores de riesgo, que no se han dado en nuestros casos, son las lesiones por aplastamiento y un tiempo prolongado de isquemia como en las amputaciones, ambos deben tenerse presentes en los pacientes con fracturas de falanges distales para evitar complicaciones. La clínica que han presentado nuestros pacientes

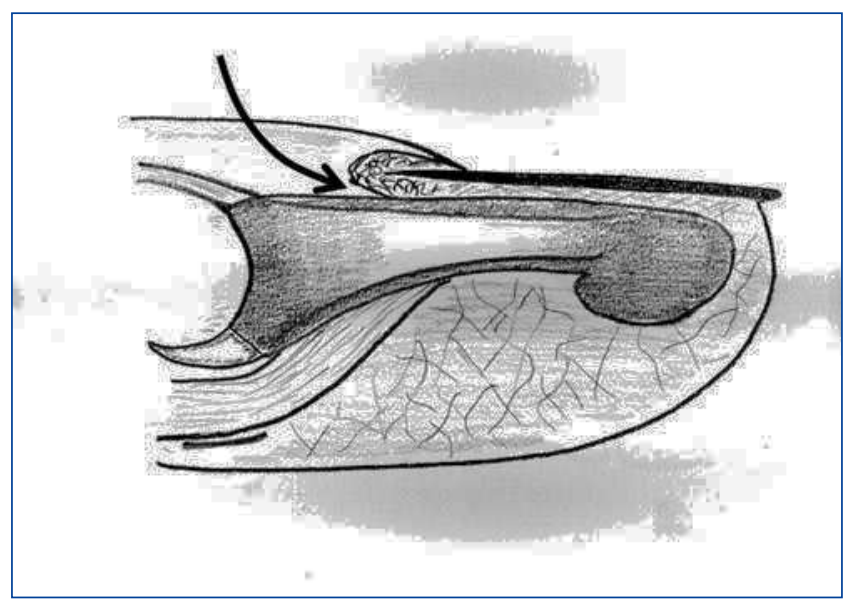

Figura 5 Esquema sagital para representar el acceso dorsal a la base de la falange distal entre la inserción del tendón extensor y la matriz ungueal. es de dificultad en las tareas que requieren realizar la pinza con fuerza. Puede además presentar una distrofia ungueal por lesión de la matriz ungueal, y disminución de la movilidad de la articulación interfalángica distal.

En los pacientes que progresan a una pseudoartrosis, el tratamiento debe encaminarse a realizar un abordaje quirúrgico que minimice el daño a los tejidos perilesionales y que, además, permita realizar una adecuada fijación. Por último, aconsejamos el aporte de injerto esponjoso.

\section{Conflicto de intereses}

Los autores declaran no tener ningún conflicto de intereses.

\section{Bibliografía}

1. Day CS, Stern PJ. Fractures of metacarpals and phalanges. En: Green PF, editor. Operative hand surgery. $6 .^{a}$ ed. New York: Churchill Livingstone; 2011. p. 258-9.

2. Jupiter JB, Koniuch MP, Smith RJ. The management of delayed union and nonunion of the metacarpals and phalanges. J Hand Surg Am. 1985;10:457-66.

3. Schneider LH. Fractures of the distal phalanx. Hand Clin. 1988;4:537-47.

4. Schneider LH. Fractures of the distal interphalangeal joint. Hand Clin. 1994;10:277-85.

5. Ring D. Malunion and nonunion of the metacarpals and phalanges. Instr Course Lect. 2006;55:121-8.

6. DaCruz DJ, Slade RJ, Malone W. Fractures of the distal phalanges. J Hand Surg [Br]. 1988;13:350-2.

7. Barton NJ. Fractures of the shafts of the phalanges of the hand. Hand. 1979;11:119-33.

8. Van Oosterom FJ, Brete GJ, Ozdemir C, Hovius SE. Treatment of phalangeal fractures in severely injured hands. J Hand Surg [Br]. 2001;26:108-11.

9. Itoh Y, Uchinishi K, Oka Y. Treatment of pseudoarthrosis of the distal phalanx with the palmar midline approach. J Hand Surg Am. 1983;8:80-4.

10. Chim H, Teoh LC, Yong FC. Open reduction and interfragmentary screw fixation for symtomatic nonunion of distal phalangeal fractures. J Hand Surg Eu Vol. 2008;33:71-6.

11. Kim J, Ki SH, Cho Y. Correction of distal phalangeal nonunion using peg bone graft. J Hand Surg Am. 2014;39:249-55.

12. Shinomiya R, Sunagawa T, Ochi M. Bone peg fixation for the treatment of nonunion of the shaft of the distal phalanx. J Hand Surg Eur Vol. 2010;35:769-71.

13. Ozçelik IB, Kabakas F, Mersa B, Purisa H, Sezer I, Ertürer E. Treatment of nonunions of the distal phalanx with olecranon bone graft. J Hand Surg Eur Vol. 2009;34:638-42.

14. Voche P, Merle M, Pillot D. [Pseudarthrosis and delayed union of the distal phalanx of the long fingers. Apropos of 13 cases]. Rev Chir Orthop Reparatrice Appar Mot. 1995;81:485-90.

15. Botelheiro JC. Treatment of pseudarthrosis of the distal phalanx with a compression screw. J Hand Surg [Br]. 1995;20:618-9. 